\title{
Gap Solitons in Periodic Discrete Nonlinear Schrödinger Equations
}

\author{
A. PANKOV \\ Mathematics Department \\ College of William and Mary \\ Williamsburg, VA 23187-8795 \\ e-mail: pankov@member .ams.org
}

\begin{abstract}
It is shown that the periodic DNLS, with cubic nonlinearity, possesses gap solutions, i. e. standing waves, with the frequency in a spectral gap, that are exponentially localized in spatial variable. The proof is based on the linking theorem in combination with periodic approximations.
\end{abstract} $78 \mathrm{~A} 40$

Mathematics subject classification: 35Q55, 35Q51, 39A12, 39A70,

\section{Introduction}

In this paper we consider spatially localized standing waves for the discrete nonlinear Schrödinger equation (DNLS)

$$
i \dot{\psi}_{n}=-\Delta \psi_{n}+\varepsilon_{n} \psi_{n}-\sigma \chi_{n}\left|\psi_{n}\right|^{2} \psi_{n}, \quad n \in \mathbb{Z},
$$

where $\sigma= \pm 1$,

$$
\Delta \psi_{n}=\left(\psi_{n+1}+\psi_{n-1}-2 \psi_{n}\right)
$$

is the discrete Laplacian in one spatial dimension and given sequences $\varepsilon_{n}$ and $\chi_{n}$ are assumed to be $N$-periodic in $n$, i. e. $\varepsilon_{n+N}=\varepsilon_{n}$ and $\chi_{n+N}=\chi_{n}$. Such solutions are often called intrinsic localized modes or breathers, but in the case under consideration we prefer the name "gap solitons" due to the obvious analogy with gap solitons in photonic crystals (see, e. g. 1, 4, 15]).

Making use the standing wave Ansatz

$$
\psi_{n}=u_{n} \exp (i \omega t)
$$

where $u_{n}$ is a real valued sequence and $\omega \in \mathbb{R}$, we arrive at the equation

$$
-\Delta u_{n}+\varepsilon_{n} u_{n}-\omega u_{n}=\sigma \chi_{n}\left|u_{n}\right|^{2} u_{n}
$$


We impose the following boundary condition at infinity:

$$
\lim _{n \rightarrow \pm \infty} u_{n}=0,
$$

and we are looking for nontrivial solutions, i. e. solutions that are not equal to 0 identically.

Actually, we consider a more general equation

$$
L u_{n}-\omega u_{n}=\sigma \chi_{n}\left|u_{n}\right|^{2} u_{n}
$$

with the same boundary condition (0.3). Here $L$ is a second order difference operator

$$
L u_{n}=a_{n} u_{n+1}+a_{n-1} u_{n-1}+b_{n} u_{n}
$$

where $a_{n}$ and $b_{n}$ are real valued $N$-periodic sequences. The operator $L$ can be represented in the form

$$
L u_{n}=-\left(\partial^{*} a_{n} \partial\right) f_{n}+\left(a_{n-1}+a_{n}+b_{n}\right) u_{n},
$$

where

$$
\partial u_{n}=u_{n+1}-u_{n}, \quad \partial^{*} u_{n}=u_{n-1}-u_{n} .
$$

When $a_{n} \equiv 1$ and $b_{n}=-2+\varepsilon_{n}$, we obtain equation (0.2).

We consider equation (0.4) as a nonlinear equation in the space $l^{2}$ of twosided infinite sequences. Note that every element of $l^{2}$ automatically satisfies (0.3).

The operator $L$ is a bounded and self-adjoint operator in $l^{2}$. Its spectrum $\sigma(L)$ has a band structure, i. e. $\sigma(L)$ is a union of a finite number of closed intervals (see, e. g., [25]). The complement $\mathbb{R} \backslash \sigma(L)$ consists of a finite number of open intervals called spectral gaps. Two of them are semi-infinite. We fix one such gap and denote it by $(\alpha, \beta)$.

Our main result is the following

Theorem 0.1 Suppose that $\chi_{n}>0$ and $\omega \in(\alpha, \beta)$. If either $\sigma=+1$ and $\beta \neq+\infty$, or $\sigma=-1$ and $\alpha \neq-\infty$, then equation (0.4) has a nontrivial solution $u \in l^{2}$ and, moreover, the solution $u$ decays exponentially at infinity:

$$
\left|u_{n}\right| \leq C e^{-\gamma|n|}, \quad n \in \mathbb{Z},
$$

with some $C>0$ and $\gamma>0$. If either $\sigma=+1$ and $\beta=+\infty$, or $\sigma=-1$ and $\alpha=-\infty$, then there is no nontrivial solution in $l^{2}$.

The proof contained in Sections 15 is variational. Its idea is borrowed from 17 and based on so-called periodic approximations. In what follows we consider the case $\sigma=+1$. The other case reduces to the previous one if we replace $L$ by $-L$ and $\omega$ by $-\omega$. 


\section{Variational setting}

On the Hilbert space $E=l^{2}$, we consider the functional

$$
J(u)=\frac{1}{2}(L u-\omega u, u)-\frac{1}{4} \sum_{n=-\infty}^{+\infty} \chi_{n} u_{n}^{4},
$$

where $(\cdot, \cdot)$ is $l^{2}$ inner product. The corresponding norm in $E$ is denoted by $\|\cdot\|$. The functional $J$ is a well-defined $C^{1}$ functional on $E$ and equation (0.4) is easily recognized as the corresponding Euler-Lagrange equation for $J$ (remind that $\sigma=+1$ ). Thus, we are looking for nonzero critical points of $J$.

Fix an integer $k>0$ and denote by $E_{k}$ the space of all $k N$-periodic sequences. This is a $k N$-dimensional Hilbert space endowed with the inner product

$$
(u, v)_{k}=\sum_{n=0}^{k N-1} u_{n} v_{n}, \quad u, v \in E_{k}
$$

and corresponding norm $\|\cdot\|_{k}$. On the space $E_{k}$ we consider the functional

$$
J_{k}(u)=\frac{1}{2}(L u-\omega u, u)_{k}-\frac{1}{4} \sum_{n=0}^{k N-1} \chi_{n} u_{n}^{4} .
$$

Due to the periodicity of coefficients the operator $L$ acts in $E_{k}$. Critical points of $J_{k}$ are exactly $k N$-periodic solutions of equation (0.4) with $\sigma=+1$.

For gradients of $J$ and $J_{k}$ we have the following formulas:

$$
(\nabla J(u), v)=(L u-\omega u, v)-\sum_{n=-\infty}^{+\infty} \chi_{n} u_{n}^{3} v_{n}, \quad v \in E,
$$

and

$$
\left(\nabla J_{k}(u), v\right)=\left(L_{k} u-\omega u, v\right)_{k}-\sum_{n=0}^{k N-1} \chi_{n} u_{n}^{3} v_{n}, \quad v \in E .
$$

We denote by $L_{k}$ the operator $L$ acting in $E_{k}$. From the spectral theory of difference (Jacobi) operators (see, e. g., 25]) it follows immediately that $\sigma\left(L_{k}\right) \subset \sigma(L)$ and, hence, $\left\|L_{k}\right\| \leq\|L\|$.

Let $E_{k}^{+}$(respectively, $E_{k}^{-}$) be the positive (respectively, negative) spectral subspace of the operator $L_{k}-\omega$ in $E_{k}$. Similarly, we introduce the positive and negative spectral subspaces $E^{+} \subset E$ and $E^{-} \subset E$, respectively, for the operator $L-\omega$. Let

$$
\delta=\min [|\alpha-\omega|,|\beta-\omega|]
$$

be the distance from $\omega$ to the spectrum $\sigma(L)$. Then

$$
\pm(L u-\omega u, u) \geq \delta\|u\|^{2}, \quad u \in E^{ \pm},
$$

and

$$
\pm\left(L_{k} u-\omega u, u\right)_{k} \geq \delta\|u\|_{k}^{2}, \quad u \in E_{k}^{ \pm} .
$$

Now we are ready to prove the nonexistence part of Theorem 0.1 
Proposition 1.1 Suppose that $\beta=+\infty$. Then the only critical point of $J$ (respectively, $J_{k}$ ) is the origin of the space $E$ (respectively, $E_{k}$ ).

Proof. We consider the case of $J$, the remaining case being similar.

Let $u \in E$ be a critical point of $J$. Then, by (1.3) and positivity of $\chi_{n}$,

$$
\begin{aligned}
0 & =(\nabla J(u), u)=(L u-\omega u, u)-\sum_{n=-\infty}^{+\infty} \chi_{n} u_{n}^{4} \leq \\
& \leq(L u-\omega u, u) .
\end{aligned}
$$

Since $\beta=+\infty$, we have that $E^{+}=\{0\}$ and, by (1.5),

$$
0 \leq-\delta\|u\|^{2}
$$

which implies that $u=0$.

\section{Technical results}

To prove the existence of $k N$-periodic solutions, as well as to pass to the limit as $k \rightarrow \infty$, we need some preliminaries. We start with

Lemma 2.1 For any nontrivial critical points $u^{(k)} \in E_{k}$ of $J_{k}$ and $u \in E$ of $J$, with critical values $c^{(k)}=J_{k}\left(u^{(k)}\right)$ and $c=J(u)$, we have

$$
\left\|u^{(k)}\right\|_{k} \leq 4 \delta^{-1} \bar{\kappa} \underline{\kappa}^{-3 / 4}\left(c^{(k)}\right)^{3 / 4}
$$

and

$$
\|u\| \leq 4 \delta^{-1} \bar{\kappa}^{-3 / 4} c^{3 / 4},
$$

where $\underline{\kappa}=\min \left\{\chi_{n}\right\}$ and $\bar{\kappa}=\max \left\{\chi_{n}\right\}$.

Proof. We have

$$
\begin{aligned}
c & =J(u)-\frac{1}{2}(\nabla J(u), u)=\left(\frac{1}{2}-\frac{1}{4}\right) \sum_{n=-\infty}^{+\infty} \chi_{n} u_{n}^{4} \geq \\
& \geq \frac{1}{4} \underline{\kappa}\|u\|_{l^{4}}^{4},
\end{aligned}
$$

where $\|\cdot\|_{l^{p}}$ stands for the norm in the space $l^{p}$. Let $u^{ \pm}$be the orthogonal projection of $u$ into $E^{ \pm}$along $E^{\mp}$. Then

$$
\begin{aligned}
0 & =\left(\nabla J(u), u^{+}\right)=\left(L u-\omega u, u^{+}\right)-\sum_{n=-\infty}^{+\infty} \chi_{n} u_{n}^{3} u_{n}^{+}= \\
& =\left(L u^{+}-\omega u^{+}, u^{+}\right)-\sum_{n=-\infty}^{+\infty} \chi_{n} u_{n}^{3} u_{n}^{+}
\end{aligned}
$$


and we obtain, using Hölder's inequality, that

$$
\begin{aligned}
\delta\left\|u^{+}\right\|^{2} & \leq \bar{\kappa}\left[\sum_{n=-\infty}^{+\infty} u_{n}^{6}\right]^{1 / 2}\left[\sum_{n=-\infty}^{+\infty}\left(u_{n}^{+}\right)^{2}\right]^{1 / 2}= \\
& =\bar{\kappa}\|u\|_{l^{6}}^{3}\left\|u^{+}\right\| \leq \bar{\kappa}\|u\|_{l^{4}}^{3}\left\|u^{+}\right\| .
\end{aligned}
$$

Hence, by (2.1),

$$
\left\|u^{+}\right\|^{2} \leq 2^{3 / 2} \delta^{-1} \underline{\kappa}^{-3 / 4} c^{3 / 4}\left\|u^{+}\right\|
$$

Similarly,

$$
\left\|u^{-}\right\|^{2} \leq 2^{3 / 2} \delta^{-1} \bar{\kappa} \underline{\kappa}^{-3 / 4} c^{3 / 4}\left\|u^{-}\right\|
$$

Since

$$
\|u\|^{2}=\left\|u^{+}\right\|^{2}+\left\|u^{-}\right\|^{2}
$$

and

$$
\left\|u^{+}\right\|+\left\|u^{-}\right\| \leq 2^{1 / 2}\|u\|
$$

the two previous inequalities imply that

$$
\|u\| \leq 4 \delta^{-1} \bar{\kappa} \underline{\kappa}^{-3 / 4} c^{3 / 4} .
$$

The remaining case of $J_{k}$ is similar.

We need also lower estimates for nontrivial critical points and critical values.

Lemma 2.2 Under the notation of Lemma 2.1

$$
\begin{gathered}
\|u\|^{2} \geq 2^{-1 / 2} \delta \bar{\kappa}^{-1}, \\
c \geq \frac{1}{8} \delta^{2} \bar{\kappa}^{-2} \underline{\kappa}, \\
\left\|u^{(k)}\right\|_{k}^{2} \geq 2^{-1 / 2} \delta \bar{\kappa}^{-1}
\end{gathered}
$$

and

$$
c^{(k)} \geq \frac{1}{8} \delta^{2} \bar{\kappa}^{-2} \underline{\kappa} .
$$

Proof. Consider the case of $J$, the other case being similar.

Since $J^{\prime}(u)=0$, then $\left(J^{\prime}(u), v\right)=0$ for any $v \in E$. Taking $v=u^{+}$, the orthogonal projection of $u$ onto $E^{+}$, we have as in the proof of Lemma 2.1

$$
\delta\left\|u^{+}\right\|^{2} \leq \sum_{n \in \mathbb{Z}} \chi_{n} u_{n}^{3} u_{n}^{+} .
$$

Using the Hölder inequality with $p=4 / 3$ and $p^{\prime}=4$, we obtain

$$
\delta\left\|u^{+}\right\| \leq \bar{\kappa}\|u\|_{l^{4}}^{3}\left\|u^{+}\right\|_{l^{4}} \leq \bar{\kappa}\|u\|^{3}\left\|u^{+}\right\| .
$$


Similarly, taking $v=u^{-}$, the orthogonal projection of $u$ onto $E^{-}$, we obtain that

$$
\delta\left\|u^{-}\right\|^{2} \leq \bar{\kappa}\|u\|^{3}\left\|u^{-}\right\| .
$$

Combining the last two inequalities, we have that

$$
\delta\|u\|^{2} \leq \bar{\kappa}\|u\|^{3}\left(\left\|u^{+}\right\|+\left\|u^{-}\right\|\right) \leq 2^{1 / 2} \bar{\kappa}\|u\|^{4} .
$$

Hence

$$
\|u\|^{2} \geq 2^{-1 / 2} \delta \bar{\kappa}^{-1}
$$

The bound for $c$ follows immediately from the last inequality and Lemma 2.1

\section{$3 \quad$ Existence of periodic solutions}

In this section we prove the existence of nontrivial $k N$-periodic solutions to equation (0.4). Actually, we show that the functional $J_{k}$ possesses a nontrivial critical point. Moreover, we derive some uniform in $k$ bounds for the critical points and corresponding critical values. The proof relies upon the standard linking theorem (see Appendix B).

Recall that a sequence $v^{(j)} \in E_{k}$ is called a Palais-Smale sequence for $J_{k}$ at level $b$ if $J_{k}\left(v^{(j)}\right) \rightarrow b$ and $J_{k}^{\prime}\left(v^{(j)}\right) \rightarrow 0$ as $j \rightarrow \infty$.

Lemma 3.1 The functional $J_{k}$ satisfies the so-called Palais-Smale condition, i. e. every Palais-Smale sequence contains a convergent subsequence.

Proof. Since the space $E_{k}$ is finite dimensional, it is enough to show that every Palais-Smale sequence is bounded. Moreover, replacing $L$ by $L+\omega_{0}$ and $\omega$ by $\omega+\omega_{0}$, with some $\omega_{0}$, we can assume that $L \gg 1$, i. e.

$$
(L v, v)_{k} \geq\|v\|_{k}^{2}, \quad v \in E_{k},
$$

and $\omega>0$.

Let $v^{(j)}$ be a Palais-Smale sequence at some level $b$. Fix $\beta \in\left(\frac{1}{4}, \frac{1}{2}\right)$. For $j$ large, we have

$$
\begin{aligned}
b+1+\beta\left\|v^{(j)}\right\|_{k} \geq & J_{k}\left(v^{(j)}\right)-\beta\left(J_{k}^{\prime}\left(v^{(j)}\right), v^{(j)}\right)= \\
= & \left(\frac{1}{2}-\beta\right)\left(L v^{(j)}, v^{(j)}\right)_{k}-\left(\frac{1}{2}-\beta\right) \omega\left\|v^{(j)}\right\|_{k}^{2}+ \\
& +\left(\beta-\frac{1}{4}\right) \sum_{n=0}^{k} \chi_{n}\left(v^{(j)}\right)^{4} \geq \\
\geq & \left(\frac{1}{2}-\beta\right)\left\|v^{(j)}\right\|_{k}^{2}-\left(\frac{1}{2}-\beta\right) \omega\left\|v^{(j)}\right\|_{k}^{2}+ \\
& +\left(\beta-\frac{1}{4}\right) \underline{\kappa}\left\|v^{(j)}\right\|_{k}^{4} .
\end{aligned}
$$


Since $a^{2} \leq K(\varepsilon)+\varepsilon a^{4}$, where $K(\varepsilon) \rightarrow \infty$ as $\varepsilon \rightarrow 0$, and $\omega>0$, we can choose $\varepsilon$ so small that the third term above absorbs the second one up to a constant. Hence

$$
b+1+\beta\left\|v^{(j)}\right\|_{k} \geq\left(\frac{1}{2}-\beta\right)\left\|v^{(j)}\right\|_{k}^{2}+C\left\|v^{(j)}\right\|_{k}^{4}-C_{0},
$$

with $C>0$ and $C_{0}>0$. This implies immediately that the sequence $\left\|v^{(j)}\right\|_{k}$ is bounded and the proof is complete.

Now let us check that the functional $J_{k}$ possesses the linking geometry (see Appendix (B), with $Y=E_{k}^{-}$and $Z=E_{k}^{+}$. Remind that we consider the case when $\beta \neq+\infty$ and, hence, $E_{k}^{+} \neq\{0\}$. Fix two constants $\varrho>r>0$ and choose $z^{k} \in E_{k}^{+}$as follows.

Let $z \in E^{+}$be an arbitrary unit vector. We set

$$
z^{k}=\frac{1}{\left\|P_{k}^{+} S_{k} z\right\|_{k}} P_{k}^{+} S_{k} z \in E_{k}^{+}
$$

(see Appendix $\mathrm{A}$ for the definition of operator $S_{k}$ ). The vector $z^{k}$ is well-defined at least for sufficiently large $k$, say, $k \geq k_{0}$. If $k<k_{0}$, we choose $z^{k}$ to be an arbitrary unit vector in $E_{k}^{+}$. Now we set

$$
S=\left\{v \in E_{k}^{+}:\|v\|_{k}=r\right\}
$$

and

$$
M=\left\{v=y+t z^{k}:\|v\|_{k} \leq \varrho, t \geq 0, y \in E_{k}^{-}\right\} .
$$

Let $M_{0}$ be the boundary of the set $M$,

$$
M_{0}=\left\{v=y+t z^{k}: y \in E_{k}^{-},\|v\|_{k}=\varrho \text { and } t \geq 0 \text {, or }\|y\|_{k} \leq \varrho \text { and } t=0\right\} \text {. }
$$

Lemma 3.2 For $\varrho>0$ large enough

$$
J_{k}(v) \leq 0, \quad v \in M_{0}
$$

while

$$
J_{k}(v) \geq \frac{\delta}{4} r^{2}
$$

provided $r^{2} \leq \bar{\kappa}^{-1} \delta$. Moreover, there exists a constant $C>0$ independent of $k$ and such that

$$
J_{k}(v) \leq C, \quad v \in M .
$$

Proof. For $v \in E_{k}^{+}$we have

$$
\begin{aligned}
J_{k}(v) & =\frac{1}{2}\left(L_{k} v-\omega v, v\right)_{k}-\frac{1}{4} \sum_{n=0}^{k N-1} \chi_{n} v_{n}^{4} \geq \\
& \geq \frac{\delta}{2}\|v\|_{k}^{2}-\frac{\bar{\kappa}}{4}\|v\|_{k}^{4} .
\end{aligned}
$$


This implies that, for $r^{2} \leq(\bar{\kappa})^{-1} \delta$,

$$
J_{k} \geq \frac{\delta}{4} r^{2} \quad \text { on } S .
$$

Now let us consider $J_{k}$ on $M$. Since $E_{k}^{ \pm}$are mutually orthogonal spectral subspaces of $L_{k}$,

$$
\begin{aligned}
J_{k}(y+t z)= & \frac{1}{2}\left(L_{k} y-\omega y, y\right)_{k}+\frac{t^{2}}{2}\left(L_{k} z^{k}-\omega z^{k}, z^{k}\right)_{k}- \\
& -\frac{1}{4} \sum_{n=0}^{k N-1} \chi_{n}\left(y_{n}+t z_{n}^{k}\right)^{4} .
\end{aligned}
$$

Hence,

$$
J_{k}(y+t z) \leq-\frac{\delta}{2}\|y\|_{k}^{2}+\frac{t^{2}}{2}\left(\left(L_{k}-\omega\right) z^{k}, z^{k}\right)_{k}-\frac{1}{4} \underline{\kappa}\left\|y+t z^{k}\right\|_{l_{k}^{4}}^{4} .
$$

Consider the subspace $X=E_{k}^{-} \oplus \mathbb{R} z^{k} \subset E_{k}$ endowed with the norm $\|\cdot\|_{l_{k}^{4}}$ (for the definition of $\|\cdot\|_{l_{k}^{p}}$ see Appendix A). The map $y+t z^{k} \mapsto t z^{k}$ is a bounded projector onto $\mathbb{R} z$. Since its norm is not less that 1 , we see that

$$
\left\|y+t z^{k}\right\|_{l_{k}^{4}} \geq\left\|t z^{k}\right\|_{l_{k}^{4}}
$$

Hence,

$$
J_{k}\left(y+t z^{k}\right) \leq-\frac{\delta}{2}\|y\|_{k}^{2}+\frac{t^{2}}{2}\left(\left(L_{k}-\omega\right) z^{k}, z^{k}\right)_{k}-\frac{1}{4} \underline{\kappa} t^{4}\left\|z^{k}\right\|_{l_{k}^{4}}^{4} .
$$

We have that

$$
\left(\left(L_{k}-\omega\right) z^{k}, z^{k}\right) \leq a_{0},
$$

where $a_{0}=\left\|L_{k}-\omega\right\|$, and, by (A.2) and Lemma A.1

$$
\lim _{k \rightarrow \infty}\left\|z^{k}\right\|_{l_{k}^{4}}^{4}=\left\|P^{+} z\right\|_{l^{4}}^{4}=\|z\|_{l^{4}}^{4} .
$$

Inequality (3.2) implies that

$$
J_{k}\left(y+t z^{k}\right) \leq-\frac{\delta}{2}\|y\|_{k}^{2}+\frac{a_{0}}{2} t^{2}-a_{1} t^{4} \leq \frac{a_{0}}{2} t^{2}-a_{1} t^{4},
$$

with some $a_{1}>0$ independent of $k$. Therefore, for all $\varrho$ large we have that $J_{k} \leq 0$ on $M_{0}$. Moreover,

$$
\sup _{M} J_{k}(v) \leq C=\max _{t>0}\left(\frac{a_{0}}{2} t^{2}-c_{1} t^{4}\right)
$$

with $C>0$ independent of $k$. The proof is complete.

Now we are ready to prove the existence of periodic solutions. Remind that, without loss of generality, we consider the case $\sigma=+1$. 
Theorem 3.1 Suppose that $\chi_{n}>0$ and $\omega \in(\alpha, \beta)$, with $\beta \neq+\infty$. Then for every $k \geq 1$ equation 0.4 ), with $\sigma=+1$, has a nontrivial $k N$-periodic solution $u^{(k)}$. Moreover, we have the following bounds:

$$
\begin{gathered}
J_{k}\left(u^{(k)}\right) \leq C, \\
\left\|u^{(k)}\right\|_{k} \leq C,
\end{gathered}
$$

where $C>0$ is independent of $k$.

Proof. The existence follows immediately from the standard linking theorem (see Appendix B). Indeed, to apply that theorem we need two things: (a) the Palais-Smale condition and (b) the linking geometry. The Palais-Smale condition is verified in Lemma 3.1] while Lemma 3.2 means exactly that the functional $J_{k}$ possesses the linking geometry.

Moreover, the linking theorem states that the corresponding critical value satisfies

$$
J_{k}\left(u^{(k)}\right) \leq \sup _{M} J_{k}(v) .
$$

Therefore, the upper bounds for the critical points and critical values follow from Lemma 3.2 and Lemma 2.1 .

\section{Existence of localized solutions}

The following result gives the existence of solution $u \in l^{2}$ in Theorem 0.1

Theorem 4.1 Under assumptions of Theorem 3.1 equation (0.4) possesses a nontrivial solution $u \in l^{2}$.

Proof. Consider the sequence $u^{(k)}=\left\{u_{n}^{(k)}\right\}$ of $k N$-periodic solutions found in Theorem 3.1

First we claim that there exists $\delta_{0}>0$ and $n_{k} \in \mathbb{Z}$ such that

$$
\left|u_{n_{k}}^{(k)}\right| \geq \delta_{0} .
$$

Indeed, if not, then $u^{(k)} \rightarrow 0$ in $l^{\infty}$. Hence, $v^{(k)}=R_{k} u^{(k)} \rightarrow 0$ in $l^{\infty}$. By Theorem 3.2 $\left\|v^{(k)}\right\|_{l^{2}}=\left\|u^{(k)}\right\|_{k}$ is bounded. Now the following simple inequality

$$
\|v\|_{l^{p}}^{p} \leq\|v\|_{l^{\infty}}^{p-2}\|v\|_{l^{2}}^{2}
$$

where $p>2$, shows that $v^{(k)} \rightarrow 0$ in all $l^{p}, p>2$. Hence, $\left\|u^{(k)}\right\|_{l_{k}^{p}} \rightarrow 0$ for all $p>2$. As in the beginning of proof of Lemma 2.1. we have that for the corresponding critical value $c^{k}=J_{k}\left(u^{(k)}\right)$

$$
0<c^{k}=\frac{1}{4} \sum_{n \in Q_{k}} \chi_{n}\left[u_{n}^{(k)}\right]^{4} \leq \frac{\bar{\kappa}}{4}\left\|u^{(k)}\right\|_{l_{k}^{4}}^{4} \rightarrow 0
$$

However, this contradicts Lemma 2.2 and we obtain (4.1). 
Due to periodicity of coefficients, $\left\{u_{n+N}^{(k)}\right\}$ is also a solution of (0.4). Hence, making such shifts, we can assume that $0 \leq n_{k} \leq N-1$ in (4.1). Moreover, passing to a subsequence of $k$ 's, we can even assume that $n_{k}=n_{0}$ is independent of $k$.

Next we extract a subsequence, still denoted by $u^{(k)}$, such that $u_{n}^{(k)} \rightarrow u_{n}$ for every $n \in \mathbb{Z}$. Inequality (4.1) implies that $\left|u_{n_{0}}\right| \geq \delta_{0}$ and, hence, $u=\left\{u_{n}\right\}$ is a nonzero sequence. It is not difficult to show that equation (0.4) possesses point-wise limits and, therefore, $u$ is a solution of that equation.

Finally we have that

$$
\sum_{n=-a}^{a}\left|u_{n}^{(k)}\right|^{2} \leq\left\|u^{(k)}\right\|_{k} \leq C
$$

for any fixed $a \in \mathbb{Z}$ and $k$ large enough. Passing to the limit, we have that

$$
\sum_{n=-a}^{a}\left|u_{n}\right|^{2} \leq C
$$

Since $a$ is arbitrary, $u \in l^{2}$.

Remark 4.1 Consider equation (0.4) with small nonlinearity, i. e.

$$
L u_{n}-\omega u_{n}=\sigma \lambda \chi_{n}\left|u_{n}\right|^{2} u_{n}
$$

where $\lambda>0$ is a small parameter (all other data, including $\omega$, are fixed). Then Lemma 2.1 shows that for the solution $u=u_{\lambda} \in l^{2}$ obtained in Theorem 0.1 we have that

$$
\left\|u_{\lambda}\right\|^{2} \geq c \lambda^{-1}
$$

with $c>0$. This means that the solution $u_{\lambda}$ bifurcates from infinity. Actually, one can scale out $\lambda$ and obtain the same conclusion directly.

\section{Exponential decay and nonexistence result}

To complete the proof of Theorem 0.1 we have to show that the solution obtained decays exponentially fast. Actually, we have

Theorem 5.1 Under assumptions of Theorem 3.1 let $u \in l^{2}$ be a solution of equation (0.4). Then $u$ satisfies

$$
\left|u_{n}\right| \leq C e^{-\gamma|n|}, \quad n \in \mathbb{Z},
$$

with some $C>0$ and $\gamma>0$.

Proof. Let $v_{n}=-\sigma \chi_{n}\left|u_{n}\right|^{2}$. Then

$$
\widetilde{L} u_{n}-\omega u_{n}=0
$$


where

$$
\widetilde{L} u_{n}=L u_{n}+v_{n} u_{n} .
$$

Since $\lim _{|n| \rightarrow \infty} v_{n}=0$, the multiplication by $v_{n}$ is a compact operator in $l^{2}$. Hence,

$$
\sigma_{e s s}(\widetilde{L})=\sigma_{e s s}(L),
$$

where $\sigma_{\text {ess }}$ stands for the essential spectrum. Now (5.1) means that $u=\left\{u_{n}\right\}$ in an eigenfunction that corresponds to the eigenvalue of finite multiplicity $\omega \notin$ $\sigma_{\text {ess }}(\widetilde{L})$ of the operator $\widetilde{L}$. Therefore, the result follows from the standard theorem on exponential decay for such eigenfunctions (see, e. g. 25]).

The following result shows that if $\omega \in \sigma(L)$, then equation (0.4) has no well-decaying (e. g., exponentially fast) nontrivial solution.

Theorem 5.2 Suppose that $\omega \in \sigma(L)$ and $u$ is a solution of (0.4) such that $|n|^{1 / 2} u_{n} \in l^{2}$. The $u_{n} \equiv 0$.

Proof. We have that $u$ satisfies (5.1) and this means that $\omega$ is an eigenvalue embedded into $\sigma_{e s s}(\widetilde{L})$. The potential $v=\left\{v_{n}\right\}$ satisfies $|n| v_{n} \in l^{1}$ and, by Theorem 7.11, 25], $\sigma_{\text {ess }}(L)$ is absolutely continuous, hence, contains no embedded eigenvalues.

\section{An extension of main result}

Consider the following equation

$$
L u_{n}-\omega u_{n}=\sigma f_{n}\left(u_{n}\right)
$$

which is more general that (0.4). Here the operator $L$ is of the same form as above, $\omega$ belongs to some spectral gap $(\alpha, \beta)$ of $L$ and $\sigma= \pm 1$. The nonlinearity $f_{n}(u)$ is supposed to satisfy the following assumptions.

(i) The function $f_{n}(u)$ is continuous in $u \in \mathbb{R}$ and depends periodically in $n$, with period $N$.

(ii) There exist $p>2$ and $c>0$ such that

$$
0 \leq f_{n}(u) \leq c|u|^{p-1}
$$

near $u=0$.

(iii) There exists $\mu>2$ such that

$$
0<\mu F_{n}(u) \equiv \int_{0}^{u} f_{n}(t) d t \leq f_{n}(u) u, \quad u \neq 0 .
$$

Arguing as in the proof of Theorem 0.1, with corresponding modifications (see 17] for a similar result for continuum periodic nonlinear Schrödinger equations), we obtain the following result. 
Theorem 6.1 Under assumptions (i)-(iii) suppose that either $\sigma=+1$ and $\beta \neq+\infty$, or $\sigma=-1$ and $\alpha \neq-\infty$. Then equation (6.1) has a nontrivial exponentially decaying solution. If either $\sigma=+1$ and $\beta=+\infty$, or $\sigma=-1$ and $\alpha=-\infty$, then there is no nontrivial solution in $l^{2}$.

The most important example is the power nonlinearity

$$
f_{n}(u)=\chi_{n}|u|^{p-2} u,
$$

with $p>2$. If $p=4$, we obtain the cubic nonlinearity considered in Theorem 0.1

Theorem 6.1 can be extended immediately to the case of equation (6.1) on $\mathbb{Z}^{d}, d \geq 1$, where

$$
L u_{n}=\sum_{m \in \mathbb{Z}^{d}} a(n, m) u_{m} .
$$

Here $a(n, m)$ satisfies

$$
a(n+N, m+N)=a(n, m)
$$

for some $N=\left(N_{1}, N_{2}, \ldots, N_{d}\right)$ and

$$
a(n, m)=0
$$

whenever $|n-m| \geq a_{0}>0$. The nonlinearity $f_{n}, n \in \mathbb{Z}^{d}$, must satisfy (i)-(iii) and the periodicity assumption

$$
f_{n+N}(u)=f_{n}(u),
$$

with the same $N$ as above.

\section{Concluding remarks}

In the past decade, localized solutions of DNLS has become a topic of intense research. Much of this work concerns the standard constant coefficient cubic DNLS and has been summarized in reviews [7, 11, 13. Constant coefficient DNLS with general power nonlinearity [6.2) $\left(\chi_{n} \equiv 1\right)$ is considered in [27].

Certainly, DNLS with periodic coefficients is not less important. In this case a new phenomenon appears. While the spectrum of $-\Delta$ consists of a single closed interval, in the spectrum of periodic operator $L$ finite gaps typically open up. The corresponding DNLS may have standing wave solutions with carrier frequency in such a gap. Theorems 0.1 and 6.1 give rigorous results of this type.

Note that in the case of periodic DNLS, and even for more general equations, with $\omega$ below or above the spectrum (depending on $\sigma$ ), such solutions are shown to exist in 21. In that paper the so-called Nehari manifold approach is employed, together with a discrete version of concentration compactness principle. For such values of $\omega$ the quadratic part of the corresponding functional is positive or negative definite. This fact simplifies the situation considerably. When $\omega$ lies in a finite gap, the quadratic part of the functional is strictly indefinite. This 
suggests us to use the linking theorem combined with periodic approximations. Such approach was used before in [16, 17, 20, 18, 19, 24]. Another approach to localized solutions of periodic DNLS, based on the generalized linking theorem (see, e. g. 28]) will be discussed elsewhere.

Note that two-dimensional discrete gap solitons are observed experimentally [8, 9]. In [10] results on numerical simulation of gap solutions in a particular periodic DNLS are reported. ${ }^{1}$

\section{A Operator $L$}

Let $l$ denote the vector spaces of all two sided complex valued sequences $u=$ $\left\{u_{n}\right\}_{n \in \mathbb{Z}}$ and $l^{p} \subset l, 1 \leq p \leq \infty$, the subspace of all $p$-summable (bounded if $p=\infty)$ sequences. Endowed with the standard norm $\|\cdot\|_{l^{p}}, l^{p}$ is a Banach spaces (Hilbert space when $p=2$ ).

Let $N \geq 1$ be a given integer and $a_{n}$ and $b_{n}$ two real valued sequences. The formula

$$
L u_{n}=a_{m} u_{n+1}+a_{n-1} u_{n-1}+b_{n} u_{n}
$$

defines a linear operator acting in the space $l$. Moreover, $L$ is a bounded linear operator in the space $l^{p}, 1 \leq p \leq \infty$, and this is a self-adjoint operator in $l^{2}$. The space $E_{k}$ of all $k N$-periodic sequences is a finite dimensional subspace of $l^{\infty}$ invariant with respect to $L$. The restriction of $L$ to $E_{k}$ is denote by $L_{k}$.

Let

$$
Q_{k}=\left\{n \in \mathbb{Z}:-\left[\frac{k N}{2}\right] \leq n \leq k N-\left[\frac{k N}{2}\right]-1\right\},
$$

where $[x]$ stands for the integer part of $x$. For a sequence $u=\left\{u_{n}\right\} \in l$ we set

$$
R_{k} u_{n}=\left\{\begin{array}{lll}
u_{n} & \text { if } & n \in Q_{k} \\
0 & \text { if } & n \notin Q_{k}
\end{array}\right.
$$

and denote by $S_{k} u_{n}$ a unique sequence that belongs to $E_{k}$ and such that

$$
S_{k} u_{n}=u_{n} \quad \text { if } n \in Q_{k} .
$$

Thus, $R_{k}$ is a "cut off" operator, while $S_{k}$ is "periodization" operator. For $u \in E_{k}$ we set

$$
\|u\|_{l_{k}^{p}}=\left\|R_{k} u\right\|_{l^{p}} .
$$

For any fixed $k,\|\cdot\|_{l_{k}^{p}}, 1 \leq p \leq \infty$, form a family of equivalent norms on $E_{k}$, $\|\cdot\|_{l_{k}^{2}}=\|\cdot\|_{k}$ is the standard Euclidean norm on $E_{k}$ and $\left(R_{k} u, R_{k} v\right)=(u, v)_{k}$ is the standard inner product on $E_{k}$.

Obviously,

$$
\left\|R_{k} u\right\|_{l^{p}} \leq\|u\|_{l^{p}}
$$

and

$$
\left\|S_{k} u\right\|_{l_{k}^{p}} \leq\|u\|_{l^{p}}
$$

\footnotetext{
${ }^{1}$ The author thanks J. Fleischer and A. Gorbach for these references.
} 
for all $u \in l^{p}$. The following identities are easy to verify: for every $u \in l^{p}$, $1 \leq p<\infty$,

$$
\begin{gathered}
\lim _{k \rightarrow \infty}\left\|R_{k} u\right\|_{l^{p}}=\lim _{k \rightarrow \infty}\left\|S_{k} u\right\|_{l_{k}^{p}}=\|u\|_{l^{p}}, \\
\lim _{k \rightarrow \infty}\left\|L R_{k} u\right\|_{l^{p}}=\lim _{k \rightarrow \infty}\left\|R_{k} L u\right\|_{l_{k}^{p}}=\|L u\|_{l^{p}}, \\
\lim _{k \rightarrow \infty}\left\|L_{k} S_{k} u\right\|_{l_{k}^{p}}=\lim _{k \rightarrow \infty}\left\|S_{k} L u\right\|_{l_{k}^{p}}=\|L u\|_{l^{p}} .
\end{gathered}
$$

Let $\lambda \in \mathbb{C} \backslash \sigma(L)$. Then the operator $L-\lambda$ is invertible in $E=l^{2}$ and for every $u=\left\{u_{n}\right\} \in l^{2}$

$$
(L-\lambda)^{-1} u_{n}=\sum_{m \in \mathbb{Z}} G(n, m ; \lambda) u_{m},
$$

where $G(\cdot, \cdot ; \lambda)$ is the so-called Green function. Due to the periodicity assumption,

$$
G(\cdot+N, \cdot+N ; \lambda)=G(\cdot, \cdot ; \lambda)
$$

(periodicity of the Green function along the diagonal). Moreover, the Green function possesses an exponential bound of the form

$$
|G(n, m ; \lambda)| \leq C e^{-\mu|m-n|}, \quad n, m \in \mathbb{Z}
$$

where $C>0$ and $\mu>0$ can be chosen independent of $\lambda$ as $\lambda$ ranges over any compact subset of $\mathbb{C} \backslash \sigma(L)$ (see, e. g. 25]). Representation (A.5) and inequality A.7 imply that for every $\lambda \in \mathbb{C} \backslash \sigma(L)$ the operator $(L-\lambda)^{-1}$ is a bounded linear operator in $l^{p}$ for every $p \in[1, \infty]$. Moreover, the space $E_{k}$ is invariant with respect to $(L-\lambda)^{-1}$ and, for every $u \in E_{k}$,

$$
\left\|\left(L_{k}-\lambda\right)^{-1} u\right\|_{l_{k}^{p}} \leq C_{p}\|u\|_{l_{k}^{p}}
$$

with $C_{p}>0$ independent of $k$. In particular, $\sigma\left(L_{k}\right) \subset \sigma(L)$.

Let $(\alpha, \beta)$ be a spectral gap of $L$. Denote by $P^{+}$and $P^{-}$the spectral projectors in $E=l^{2}$ that correspond to the parts of $\sigma(L)$ lying in $(-\infty, \alpha]$ and $[\beta,+\infty)$, respectively. Note that these are orthogonal projectors. The associated spectral subspaces are denoted by $E^{+}$and $E^{-}$, respectively. Also, we note that $\sigma\left(L_{k}\right) \cap(\alpha, \beta)=\emptyset$. We denote by $P_{k}^{+}$and $P_{k}^{-}$the spectral projectors in $E_{k}$ that correspond to $\sigma\left(L_{k}\right) \cap(-\infty, \alpha]$ and $\sigma\left(L_{k}\right) \cap[\beta,+\infty)$, respectively, and set $E_{k}^{ \pm}=P_{k}^{ \pm} E_{k}$ for the spectral subspaces.

The projectors $P^{ \pm}$are given by the Riesz formula

$$
P \pm=-\frac{1}{2 \pi i} \int_{C_{ \pm}}(L-\lambda)^{-1} d \lambda,
$$

where $C_{+}$(resp., $C_{-}$) is a contour in $\mathbb{C} \backslash \sigma(L)$ encircling $\sigma(L) \cap[\beta,+\infty$ ) (resp., $\sigma(L) \cap(-\infty, \alpha])$ so that $\sigma(L) \cap(-\infty, \alpha]$ (resp., $\sigma(L) \cap[\beta,+\infty)$ ) lies outside. Therefore, $P^{ \pm}$is of the form

$$
P^{ \pm} u_{n}=\sum_{m \in \mathbb{Z}} K(m, n) u_{m},
$$


where

$$
K(m, n)=-\frac{1}{2 \pi i} \int_{C_{ \pm}} G(m, n ; \lambda) d \lambda .
$$

Hence

$$
|K(m, n)| \leq C e^{-\mu|m-n|}, \quad m, n \in \mathbb{Z},
$$

with some $C>0$ and $\mu>0$. Note that the right hand part of (A.8) is a bounded linear operator in all spaces $l^{p}, 1 \leq p \leq \infty$. Moreover, the operators $P_{k}^{ \pm}$are exactly the restrictions of $P^{ \pm}$from $l^{\infty}$ to $E_{k}$ and, therefore, are given by the same formula A.8. Also it is easy to verify the operator

$$
B=(L-\omega) P^{ \pm}=P^{ \pm}(L-\omega)
$$

has a representation of the form

$$
B u_{n}=\sum_{m \in \mathbb{Z}} K(m, n) u_{m},
$$

with $K(m, n)$ (not the same as in A.8) satisfying A.9.

Lemma A.1 Let $B$ be an operator of the form A.10 satisfying A.9. Then for any $u \in l^{2}$

$$
\lim _{k \rightarrow \infty}\left(B S_{k} u, S_{k} u\right)_{k}=(B u, u) .
$$

The proof is contained in the proof of Theorem 3, 5 .

\section{B Linking}

Here we recall the so-called linking theorem (see 23, 28). Let $X=Y \oplus Z$ be a Banach space decomposed into the direct sum of two closed subspaces $Y$ and $Z$, with $\operatorname{dim} Y<\infty$. Let $\varrho>r>0$ and let $z \in Z$ be a fixed vector, $\|z\|=1$. Define

$$
M=\{u=y+\lambda z: y \in Y,\|u\| \leq \varrho, \lambda \geq 0\}
$$

and

$$
N=\{u \in Z:\|u\|=r\} .
$$

Denote by $M_{0}=\partial M$ the boundary of $M$, i. e.

$$
M_{0}=\{u=y+\lambda z: y \in Y,\|u\|=\varrho \text { and } \lambda \geq 0 \text {, or }\|u\| \leq \varrho \text { and } \lambda=0\} .
$$

Consider a $C^{1}$ functional $\varphi$ on $E$ and suppose that $\varphi$ satisfies the PalaisSmale condition, i. e. any sequence $u^{(j)} \in E$ such that $\varphi\left(u^{(j)}\right)$ is convergent and $\varphi^{\prime}\left(u^{(j)}\right) \rightarrow 0$ contains a convergent subsequence. Suppose also that

$$
\beta=\inf _{u \in N} \varphi(u)>\alpha=\sup _{u \in M_{0}} \varphi(u) .
$$

The last assumption means that $\varphi$ possesses the so-called linking geometry. 
Let

$$
\Gamma=\left\{\gamma \in C(M ; E): \gamma=\text { id on } M_{0}\right\}
$$

Then

$$
c=\inf _{\gamma \in \Gamma} \sup _{u \in M} \varphi(\gamma(u))
$$

is a critical value of $\varphi$ and

$$
\beta \leq c \leq \sup _{u \in M} \varphi(u)
$$

\section{References}

[1] Aceves A B 2000 Optical gap solutions: Past, persent, and future; theory and experiments Chaos $10584-589$

[2] Alfimov G L, Brazhnyi V A and Konotop V V 2004 On classification of intrinsic localized modes for the discrete nonlinear Schrödinger equation Physica D 194 127-150

[3] Bishop A R, Kalosakas G, Rasmussen K Ø and Kevrekidis P G 2003 Localization in physical systems described by discrete nonlinear Schrödinger-type equations Chaos 13 588-595

[4] Bronski J C, Segev M and Weinstein M I 2001 Mathematical frontiers in optical solitons Proc. Nat. Acad. Sci. USA 98 12872-12873

[5] Bruno G, Pankov A and Tverdokhleb Y 2001 On almost-periodic operators in the spaces of sequences Acta Appl. Math. 65 153-167

[6] de Sterke C M and Sipe J E 1994 Gap Solitons Progress in Optics vol 33 (Ed. E Wolf) (Amsterdam: North-Holland) 203-260

[7] Flash S and Willis C R 1998 Discrete breathers Phys. Repts 295 181-264

[8] Fleischer J W, Carton T, Segev M, Efremidis N K and Christodoulides D N 2003 Observation of discrete solitons in optically-induced real-time waveguide arrows Phys. Rew. Letts 90023902

[9] Fleischer J W, Segev M, Efremidis N K and Christodoulides D N 2003 Observation of $2 D$ discrete solitons in optocally-induced nonlineare photonic lattices Nature 422 147-150

[10] Gorbach A and Jonasson M 2004 Gap and out-gap breathers in a binary modulated discrete nonlinear Schrödinger model Eur Phys. J. D 29 77-93

[11] Hennig D and Tsironis G P 1999 Wave transmission in nonlinear lattices Physics Repts 309 333-432

[12] Joanopoulous J D, Maede R D and Winn J N 1995 Photonic Crystals, Molding the Flow of Light (NJ: Princeton Univ. Press) 
[13] Kevrekides P G, Rasmussen KØ and Bishop A R 2001 The discrete nonlinear Schrödinger equation: a survey of recent results Intern. J. Modern Phys. B 15 2833-2900

[14] MacKay R S and Aubry S 1994 Proof of existence of breathers for timereversible or Hamiltonian netwoks of weakly coupled oscillators Nonlinearity 7 1623-1643

[15] Mills D L 1998 Nonlinear Optics. Basic Concepts (Berlin: Springer)

[16] Pankov A 2005 Travelling Waves and Periodic Oscillations in Fermi-PastaUlam Latticies (London: Imperial College Press)

[17] Pankov A to appear Periodic Nonlinear Schrödinger Equation with an Application to Photonic Crystals Milan J. Math. (Preprint version: arXiv: math. AP/0404450)

[18] Pankov A and Pflüger K 1998 On semilinear Schrödinger equaiton with periodic potential Nonlin. Anal. 33 593-609

[19] Pankov A and Pflüger K 1999 Periodic and solitary travelling waves for the generalized Kadomtsev-Petviashvili equations Math. Meth. Appl. Sci. 22 $733-752$

[20] Pankov A and Pflüger K 2000 On ground travelling waves for the generalized Kadomtsev-Petviashvili equations Math. Phys., Anal., Geom. 3 33-47

[21] Pankov A and Zakharchenko N 2000 On some discrete variational problems Acta Appl. Math. 65 295-303

[22] Pelinovsky D E, Sukhorukov A A and Kivshar Y S 2004 Bifurcations and Stability of Gap Solitons in Periodic Potentials arXiv: nlin PS/0405019

[23] Rabinowitz P H 1968 Minimax Methods in Critical Point Theory with Applications to Differential Equations (Providence, R. I.: Amer. Math. Soc.)

[24] Rabinowitz P H 1991 A note on semilinear elliptic equations on $\mathbb{R}^{n}$ Nonlinear Analysis: A Tribute in Honnour of G. Prodi, Quad. Scu. Norm. Super. Pisa 307-318

[25] Teschl G 2000 Jacobi Operators and Complletely Integrable Nonlonear Lattices (Providence, R. I.: Amer. Math. Soc.)

[26] Trombetti A, Smerzi A and Bishop A R 2003 Discrete nonlinear Schrödinger equation with defects Phys. Rev. E 67016607

[27] Weinstein M I 1999 Excitation thresholds for nonlinear localized modes on lattices Nonlinearity 12 673-691

[28] Willem M 1996 Minimax Methods (Boston: Bikhäuser) 